\title{
Eficacia del neurofeedback para el tratamiento de los trastornos del espectro autista: Una revisión sistemática
}

\author{
Estefanía García-Berjillos, Marta Aliño, Marién Gadea, Raúl Espert y Alicia Salvador \\ Universidad de Valencia, Valencia, España
}

\begin{abstract}
Resumen: Los trastornos del espectro autista (TEA) se caracterizan por déficits en la comunicación e interacción con los demás, patrones estereotipados de conducta e intereses restringidos. Presentan patrones de encefalograma (EEG) anómalos que podrían explicar estos déficits, y por ello el neurofeedback se plantea como una alternativa terapéutica dirigida a normalizarlos. El objetivo consistió en analizar el grado de eficacia del neurofeedback como tratamiento de los TEA. Se llevó a cabo una revisión sistemática de 17 estudios empíricos localizados mediante búsqueda bibliográfica exhaustiva en PsycINFO, PsycArticles y Pubmed. Los resultados indican cierta eficacia del neurofeedback para tratar los patrones anómalos de EEG y los definitorios del TEA, además de otros como déficits en atención y funciones ejecutivas, ansiedad o problemas de conducta. El neurofeedback puede considerarse un tratamiento "con apoyo experimental modesto" o "probablemente eficaz" con "apoyo controvertido", aunque se necesitan estudios metodológicamente rigurosos para determinar con mayor certeza su eficacia terapéutica.
\end{abstract}

Palabras clava: Trastornos del espectro autista; autismo; tratamiento; EEG-biofeedback; neurofeedback.

The efficacy of neurofeedback for the treatment of autism spectrum disorders: A systematic review

\begin{abstract}
Autism spectrum disorders (ASDs) are characterized by impairments in communication and interaction skills, stereotyped patterns of behavior and restricted interests. They show anomalous electroencephalographic (EEG) patterns that might explain those impairments. Neurofeedback is considered to be a therapeutic alternative for their normalization. The objective was to review the evidence on the efficacy of neurofeedback as a treatment for ASDs. We conducted a systematic review of 17 empirical studies localized thru an exhaustive bibliographic search of the databases PsycInfo, PsycArticles and Pubmed. The results indicate certain efficacy of neurofeedback in the treatment of abnormal EEG patterns and core ASD symptoms, as well as others such as impairments in attention and cognitive functions, anxiety or behavioral disorders. Neurofeedback may be considered a treatment "with modest experimental support" or "probably efficacious" with "controversial support", though more methodologically rigorous studies are needed to determine its therapeutic efficacy with more certainty.
\end{abstract}

Keywords: Autism spectrum disorders; autism; treatment; EEG-biofeedback; neurofeedback.

\section{Introducción}

Los trastornos del espectro autista (TEA) se caracterizan por la presencia de déficits en comunicación social e interacción, así como de patrones estereotipados de conducta, y de actividades o intereses restringidos. Los síntomas están presentes desde la infancia y persisten

Recibido: 31 octubre 2014; aceptado: 30 enero 2015.

Correspondencia: Marién Gadea, Departamento de Psicobiología, Facultad de Psicología, Universidad de Valencia, Avda. Blasco Ibáñez 21,46010 Valencia, España. Correo-e: marien.gadea@uv.es toda la vida, pudiendo variar su gravedad y grado de interferencia en el desarrollo. Además, pueden incluir otros síntomas como déficits en el desarrollo del lenguaje o hipersensibilidad a los estímulos sensoriales (Asociación Americana de Psiquiatría, 2013). Su prevalencia en los países desarrollados se estima alrededor de 60/10000 habitantes (Newschaffer et al., 2007), aunque estudios recientes muestran tasas más altas en algunos países como USA, donde los CDC (2012) estiman una prevalencia de 1/88 habitantes. Según una revisión acerca de los datos epidemiológicos de los TEA publicada en España en 2005 (Posada-De la Paz, Ferrari-Arroyo, Tou- 
riño-Aguilera y Boada-Muñoz, 2005), los datos de incidencia y prevalencia del trastorno en nuestro país no son bien conocidos, dada la ausencia de estudios poblacionales comprehensivos y rigurosos.

Muchos estudios han tratado de describir los sustratos neurológicos de los TEA empleando técnicas de neuroimagen o neurofisiológicas, entre las cuales el EEG es una de las más extendidas (Billeci et al., 2013; Palau-Baduell, Valls-Santasusana, Salvado-Salvado y Clofent-Torrento, 2013). Particularmente, los estudios realizados en población con TEA empleando EEG han encontrado irregularidades y anomalías en sus patrones de actividad cerebral, entre las cuales destacan una reducción de ondas Alpha (Chan, Sze y Cheung, 2007), un exceso de ondas theta y delta (Cantor, Thatcher, Hrybyk y Kaye, 1986; Chan et al., 2007; Cornew, Roberts, Blaskey y Edgar, 2012), así como de ondas Gamma (Luschekina, Podreznaya, Luschekin y Streelets, 2012; Orekhova et al., 2007) y un ligero exceso en las ondas beta, a pesar de que el cociente theta/beta se mantiene por encima de los límites de la normalidad dado que el exceso de theta es más pronunciado (Chan y Leung, 2012; Coben, Clarke, Hudspeth y Barry, 2008a). Además, se encuentran patrones atípicos de simetría-asimetría (Burnette, Henderson, Inge, Zahka, Schwartz y Mundy, 2011), especialmente para las ondas alfa y en áreas frontales (Luschekina et al., 2012), que pueden hallarse desde edades muy tempranas y servir como predictores de riesgo de padecer el trastorno (Gabard-Durnam, Tierney, Vogel-Farley, Tager-Flusberg y Nelson, 2013). Por otra parte, las personas con TEA parecen presentar un exceso de coherencia generalizada (Cantor et al., 1986) junto con patrones de coherencia reducida entre áreas cerebrales especializadas. Esta aparente contradicción se ha interpretado recientemente como un patrón de funcionamiento cerebral en el que se combinan una hipoconectividad distal junto a una hiperconectividad local, especialmente en el lóbulo frontal (Rippon, Brock, Brown y Boucher, 2007). Estas anormalidades podrían estar asociadas a déficits en la integración de información y las funciones ejecutivas, que a su vez podrían tener un impacto negativo en el funcionamiento social y cognitivo (Coben y Myers, 2008b). Se observan también anomalías en los niveles de ondas $\mathrm{Mu}$ y déficits en la supresión de las mismas, que se consideran indicadores del funcionamiento deficitario de la red de neuronas espejo (Bernier, Dawson, Webb y Murias, 2007; Oberman et al., 2005, 2013). Por último, diversos estudios han observado la presencia de descargas epileptiformes, especialmente en áreas temporales bilaterales, que podrían estar relacionadas con déficits sociales y del lenguaje (Chez et al., 2006; Yasuhara, 2010).
Así pues, a pesar de su variabilidad, todos estos hallazgos apoyan la existencia de patrones atípicos de actividad cerebral característicos de los TEA que podrían permitir incluso la diferenciación entre personas con desarrollo neurotípico y personas con distintos trastornos dentro del espectro autista (Chan et al., 2007; Dawson, Klinger, Panagiotides, Lewy y Castelloe, 1995), y que parecen relacionados con la sintomatología predominante de estos trastornos (Dorembaum, Mencel, Blume, y Fishman, 1987; Hughes y John, 1999).

Con todo lo dicho, podría considerarse la modificación de los patrones atípicos de actividad cerebral presentes en los TEA como un objetivo prioritario de las intervenciones, que podría conseguirse gracias a técnicas de neuromodulación. Otros datos que parecen apoyar esta hipótesis son, por ejemplo, el hecho de que los tratamientos cognitivo-conductuales efectivos en la reducción de la sintomatología produzcan también cambios en los patrones de EEG en personas con TEA (Dawson et al., 2012); o la efectividad que este tipo de tratamiento ha demostrado ya en diversos trastornos (Coben y Myers, 2010b) que además presentan comorbilidad sintomática con los TEA, como el trastorno de déficit de atención e hiperactividad (TDAH) (Thompson, Thompson y Reid, 2010a) o la epilepsia (Yasuhara, 2010).

El término "neuromodulación" se refiere a la capacidad de modificar el funcionamiento e incluso la estructura del sistema nervioso central. Los avances en investigación acerca de la plasticidad del sistema nervioso han llevado al desarrollo de técnicas que, basadas en esta capacidad, nos permiten influir en él y reparar posibles disfunciones. Actualmente disponemos de técnicas de neuromodulación exógenas y endógenas, las primeras suponen la aplicación de una estimulación externa que influye y modifica la actividad eléctrica cerebral, como sucede con la estimulación magnética transcraneal; por su parte, las técnicas endógenas se apoyan en la capacidad de la persona para modular su actividad cerebral normalizando sus patrones de activación y conectividad, siendo el neurofeeback (i.e. EEG-Biofeedback) una de las más extendidas (Coben y Evans, 2011a). Este trabajo se centra en el neurofeeback por tratarse de una técnica indolora y no invasiva, con escasos o ausentes efectos secundarios muy poco dañinos, con un coste relativamente bajo y con una gran versatilidad que permite adaptarlo a distintos tipos de pacientes y con distintos fines.

El neurofeeback se basa en tres supuestos: en primer lugar, se asume que los parámetros anormales de EEG reflejan la disfunción cerebral subyacente a cada trastorno, por otra parte que las personas pueden modular voluntariamente el estado o funcionamiento de su cerebro y cambiar así los parámetros anormales de EEG; y, por 
último, que el cerebro puede "memorizar" ese nuevo estado y generalizarlo a otros ámbitos (Kropotov, 2009). En condiciones normales, las personas no podemos influir sobre nuestros patrones de EEG porque no somos conscientes de ellos, no obstante, si tuviéramos información a tiempo real de nuestro funcionamiento cerebral, sí que podríamos hacerlo (Hammond, 2007). El neurofeeback da este tipo de información a los pacientes y, para facilitar el aprendizaje, se apoya en principios de condicionamiento operante (cuando la persona alcanza el patrón de EEG deseado recibe refuerzos positivos, como la continuidad en el visionado de un vídeo o película o el logro de mejores resultados en un videojuego). Así pues, el procedimiento en una sesión típica de neurofeeback es el siguiente: se colocan los electrodos de registro en diversas áreas del cráneo del paciente, la información se transfiere a un equipo que realiza las operaciones necesarias para descomponer el trazado en bandas de frecuencia y compara al momento los parámetros registrados con los que se desea alcanzar; a continuación, el paciente recibe la información sobre su funcionamiento cerebral a tiempo real, acompañada de refuerzo positivo si ésta se ajusta a los parámetros deseados. Más concretamente, en el caso de población con TEA, los protocolos de entrenamiento más empleados buscan paliar el exceso de ondas lentas, principalmente theta, e incrementar la presencia de ondas rápidas como beta o SMR, generalmente en áreas parietales y temporales; o bien normalizar los patrones atípicos de hiper- o hipo-coherencia sobre todo en áreas frontales y temporales. No obstante, en cuanto a la elección del protocolo de neurofeeback adecuado, existen estudios que muestran una mayor eficacia de los protocolos individualizados, basados una evaluación de EEG previa (Coben et al., 2010b). Por lo que respecta a la frecuencia y duración de las sesiones, lo más común es realizar 2 sesiones semanales de 30 minutos, y un total de entre 30 y 60 sesiones. Al principio los cambios no son duraderos, pero se van convirtiendo en permanentes de forma gradual (Hammond, 2007), gracias a los mecanismos de neuroplasticidad, neuromodulación y potenciación a largo plazo (Abarbanel, 1999). Algunos de los efectos secundarios que puede producir el neurofeeback son fatiga, dolores de cabeza, ansiedad, irritabilidad, etc. Son poco frecuentes, leves y pasajeros, pero es importante considerarlos y tomar medidas para evitarlos y asegurar la mayor efectividad del tratamiento. Algunas precauciones básicas que pueden ser útiles son una correcta evaluación previa, tanto a nivel cognitivo-conductual como a nivel electrofisiológico, y un tratamiento bien diseñado, con objetivos y métodos adaptados al paciente (Hammond, 2007), así como un control minucioso de los artefactos e inter- ferencias que pueden contaminar tanto la evaluación como el progreso del tratamiento (Cantor, 1999).

De acuerdo con todo lo dicho, el objetivo del presente trabajo es realizar una revisión sistemática de la evidencia disponible acerca de la eficacia del neurofeeback para el tratamiento de los TEA, sintetizando los resultados de todos estudios los empíricos al respecto. Cabe destacar que no existe actualmente ningún trabajo de este tipo publicado en lengua castellana y que, de entre aquellos en inglés, tampoco ninguno abarca todas las investigaciones. Se pretende, por tanto, determinar si este tratamiento puede considerarse o no apropiado para dicha población: si es eficaz y en qué medida, el tipo de resultados que se obtienen, las posibles variables moduladoras que pudieran influir en ellos y, por último, si la investigación disponible es suficiente o, en caso de no serlo, cuáles son sus carencias.

\section{Método}

La búsqueda bibliográfica se ha llevado a cabo de forma sistemática a través de las bases de datos PsycInfo, PsycArticles y Pubmed, empleando la misma combinación de palabras clave en cada una de ellas: (autis* AND ("Neuro feedback" OR "eeg-biofeedback" OR neurofeedback)), pudiendo contener dichos términos en cualquier campo de la publicación. El único límite de búsqueda empleado en un primer momento ha sido el idioma, de modo que solo se incluyen publicaciones en castellano e inglés. No se consideró oportuno establecer restricciones según las fechas de publicación ni la metodología empleada en los estudios dada la escasez de trabajos empíricos disponibles acerca de neurofeeback y a su relativa novedad; en este paso se seleccionaron 127 registros. Posteriormente, ya en un proceso de selección manual, se han considerado otros criterios de inclusión-exclusión para filtrar los resultados ajustados a las necesidades de la revisión, a saber: se descartan los que no se centran en población con TEA (p.ej., TDAH, trastornos del aprendizaje, etc.) y los que emplean cualquier técnica de neuro- o biofeedback que no se base en el electroencefalograma (p.ej., hemoencefalograma, magnetoencefalografía, electromiografía, SC-biofeedback, etc.), se seleccionan tanto publicaciones científicas de carácter empírico (p.ej., revisiones de la literatura, estudios de casos, estudios experimentales) como de conceptualización (p.ej., artículos, manuales especializados, etc.) y se excluyen documentos divulgativos y en formato de disertación. Con todo ello se reduce el número de trabajos de 127 publicaciones iniciales (sin contar los duplicados) a 22 seleccionadas. Entre estas se encuentran 17 empíricas y el resto de revisión, centrándose este 
trabajo en las 17 publicaciones empíricas. Esta primera etapa de la búsqueda bibliográfica se llevó a cabo en el mes de diciembre de 2013, si bien continuaron revisándose las bases de datos durante los meses subsiguientes y hasta la fecha de finalización de la revisión, en mayo de 2014, no se encontraron nuevas publicaciones.

Por otra parte, se han empleado las mismas bases de datos con otra combinación de palabras clave para localizar gran parte de los artículos de conceptualización relativos a los sustratos neurológicos de los TEA, más concretamente a los patrones de EEG característicos de los mismos. Los términos de búsqueda empleados en esta segunda búsqueda fueron: (autis* AND (EEG NOT ("Neuro feedback" OR "eeg-biofeedback" OR neurofeedback))), con la misma restricción de idioma empleada anteriormente. Entre todos los resultados obtenidos en esta búsqueda, se seleccionaron los 3 artículos de revisión más recientes, que posteriormente se complementaron con una búsqueda manual de otros 9 trabajos, extraídos mayoritariamente de sus bibliografías. Por último, a medida que se analizaba la información disponible y de acuerdo a las necesidades de la revisión, se fue ampliando la documentación empleada mediante el rastreo de la bibliografía contenida en las publicaciones trabajadas y la búsqueda de manuales adicionales; añadiendo un total de 34 documentos que, junto a los 22 seleccionados anteriormente, constituyen la bibliografía completa de 56 documentos examinados aunque la revisión propiamente dicha del presente estudio se basó en los 17 trabajos empíricos encontrados. (Véase la Figura 1).

Para evaluar el riesgo de sesgo en los estudios se atendió tanto al tipo de diseño utilizado, como al número de sujetos que compone la muestra y a los instrumentos de evaluación empleados. En este sentido, las principales amenazas a la validez encontradas hacen referencia a la posible presencia de sesgos de selección (en algunos estudios que ocultan método de asignación a los grupos o carecen de aleatorización) y de otros sesgos introducidos por el evaluador (en ciertos estudios que no emplean evaluadores ciegos o métodos de evaluación objetivos estandarizados). No obstante, cabe destacar que también encontramos bastantes investigaciones con metodologías más rigurosas, con un menor riesgo de sesgo y que aseguran una mayor validez, cuyos resultados son en su mayoría coherentes con los de aquellos estudios de menor rigor, aportándoles así un valor añadido. En cualquier caso, y aunque ningún artículo ha sido descartado de la revisión dada la escasez de investigaciones disponibles, los resultados se muestran contextualizados según el tipo de estudios de los que proceden, con sus ventajas y limitaciones, evitando así sesgar la información en la medida de lo posible.

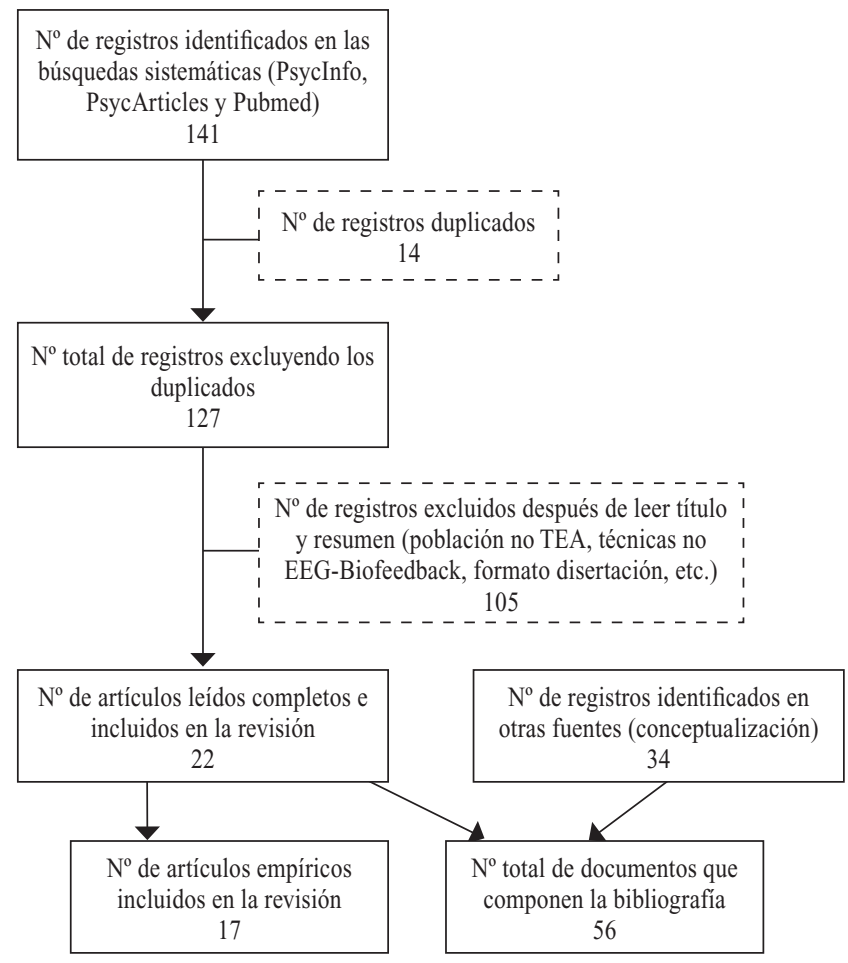

Figura 1. Diagrama de flujo mostrando el número de estudios cribados así como las razones para su exclusión.

Por lo que respecta a la extracción y análisis de los resultados, se ha empleado una metodología de tipo cualitativo. Para ello se ha realizado una síntesis de las principales características y resultados de los 17 estudios revisados (Tabla 1), que posteriormente se han organizado por áreas de interés para facilitar el análisis conjunto de la información y la elaboración de conclusiones.

\section{Resultados}

A través de la estrategia de búsqueda previamente detallada, se localizaron 17 estudios que constituyen la totalidad de los trabajos empíricos disponibles hasta el momento acerca de la eficacia del neurofeeback en el tratamiento de los TEA, y que son objeto de revisión. Entre ellos se encuentran 3 estudios de caso único, 4 series de casos y 10 estudios controlados, 3 de los cuales poseen una gran rigurosidad metodológica empleando aleatorización, placebo y diseños ciego o doble ciego.

\section{Cambios en el EEG}

De los 17 estudios revisados, 12 toman medidas de EEG como variable dependiente, y 11 de ellos muestran diferencias significativas entre las medidas de EEG prey post-tratamiento. 
Uno de los principales objetivos perseguidos en los estudios con respecto a la modificación del EEG mediante el tratamiento con neurofeeback se refiere a la reducción de ondas lentas, especialmente theta, y el incremento de ondas rápidas, generalmente beta o SMR, aunque también alfa en algunos casos. Ibric y Hudspeth (2003) fueron los primeros en presentar efectos de este tipo tras aplicar el entrenamiento con neurofeeback, que además aparecieron asociados a mejoras generalizadas en los patrones de conectividad y en la sintomatología del trastorno; resultados que Linden (2004) replica años más tarde en una pequeña serie de casos, empleando para ello protocolos individualizados (EEG-guided) (Coben, Linden y Myers, 2010a). Si bien, como puede observarse en la Tabla 1, la metodología de los trabajos previamente mencionados permite un escaso grado de certeza y generalización de resultados, existen otros trabajos más amplios y metodológicamente rigurosos que siguen la misma línea. Así, tanto dos extensas series de casos publicadas por Thompson et al. (2003; 2010b), empleando protocolos individualizados de entrenamiento, como un primer estudio controlado llevado a cabo por Kouijzer, de Moor, Gerrits, Congedo y van Schie (2009b) encuentran cambios hacia la normalización en al menos uno de los parámetros de EEG que se pretendía modificar (p.ej., reducción de theta o delta, incremento de beta o SMR). Un año después del trabajo anterior, Kouijzer, van Schie, de Moor, Gerrits y Buitelaar (2010) presentan un segundo estudio controlado en el que amplían el tamaño y la representatividad de la muestra, y registran el EEG en distintas condiciones: ojos abiertos, ojos cerrados y en movimiento; encontrando resultados significativos, no presentes en el grupo control, solo en la condición "movimiento".

Otro protocolo ampliamente aplicado a personas con TEA se centra en la regulación de las ondas $\mathrm{Mu}$, la reducción de sus niveles basales y el entrenamiento de su supresión en condiciones de observación e imitación de movimiento. Coben y Hudspeth (2006) presentaron uno de los primeros estudios controlados que muestra resultados positivos a este respecto, en el comparan la eficacia de un entrenamiento interhemisférico con un montaje bipolar y un entrenamiento de coherencia dirigido a aumentar la conectividad entre áreas centrales y periféricas del córtex frontal. Los resultados muestran una reducción significativa de Mu solo en el grupo de entrenamiento de la coherencia, asociada a mejoras en funcionamiento social (Coben y Wagner, 2011b). En la misma dirección, Pineda et al. (2008) publican dos estudios con una metodología considerablemente más rigu-

Tabla 1. Resumen de los estudios

\begin{tabular}{|c|c|c|c|c|c|}
\hline Autor & Diseño & Muestra & Tratamiento & Evaluaciones & Resultados \\
\hline $\begin{array}{l}\text { Cowan y } \\
\text { Markham } \\
(1994)\end{array}$ & Caso único. & $\begin{array}{l}\text { Chica, } 8 \text { años. Autismo } \\
\text { de alto funcionamiento. }\end{array}$ & $\begin{array}{l}21 \text { sesiones NF: } \\
\text { suprimir theta y } \\
\text { alpha-aumentar beta en } \\
\text { Pz. }\end{array}$ & $\begin{array}{l}\text { Valoración parental y de } \\
\text { profesores. TOVA. }\end{array}$ & $\begin{array}{l}\text { Mejoras en atención, } \\
\text { socialización y reducción de } \\
\text { conductas estereotipadas } \\
\text { (informe de padres y profesores). } \\
\text { Mejora de puntuaciones TOVA } \\
\text { (inatención, impulsividad y } \\
\text { variabilidad). }\end{array}$ \\
\hline $\begin{array}{l}\text { Sichel et al. } \\
\text { (1995) }\end{array}$ & Caso único. & $\begin{array}{l}\text { Chico, } 8 \text { '5 años. } \\
\text { Autismo de alto/medio } \\
\text { funcionamiento y } \\
\text { Déficit de Atención } \\
\text { (TDA). }\end{array}$ & $\begin{array}{l}37 \text { sesiones NF: reducir } \\
\text { theta-aumentar SMR en } \\
\text { Lóbulo Parietal. }\end{array}$ & $\begin{array}{l}\text { Valoración parental y de } \\
\text { su cuidadora. }\end{array}$ & $\begin{array}{l}\text { Mejoras en comunicación y } \\
\text { lenguaje, interacción, expresión y } \\
\text { comprensión emocional, } \\
\text { reducción de ansiedad y } \\
\text { conductas estereotipadas. }\end{array}$ \\
\hline $\begin{array}{l}\text { Thompson y } \\
\text { Thompson } \\
\text { (1995) }\end{array}$ & Serie de casos. & $\begin{array}{l}3 \text { casos. Diagnóstico: } \\
\text { Autismo o Asperger. }\end{array}$ & $\begin{array}{l}\text { NF: reducir theta- } \\
\text { aumentar SMR en } \mathrm{P} 4 \mathrm{y} \\
\text { T4. }\end{array}$ & - & $\begin{array}{l}\text { Mejoras en comunicación y } \\
\text { problemas de conducta. }\end{array}$ \\
\hline $\begin{array}{l}\text { Jarusiewicz } \\
(2002)\end{array}$ & $\begin{array}{l}\text { Grupo control (lista de } \\
\text { espera). Método de } \\
\text { asignación no } \\
\text { especificado. }\end{array}$ & $\begin{array}{l}\mathrm{N}=24 \text { ( } 12 \text { control-12 } \\
\mathrm{NF})\end{array}$ & $\begin{array}{l}\text { 20-69 sesiones NF: } \\
\text { protocolo adaptado a } \\
\text { QEEG previo y } \\
\text { síntomas (assesement } \\
\text { guided) }\end{array}$ & $\begin{array}{l}\text { Valoración parental. } \\
\text { ATEC. }\end{array}$ & $\begin{array}{l}\text { Reducción de } 26 \% \text { en la } \\
\text { puntuación de ATEC (vs. } 3 \% \text { en } \\
\text { grupo control). Mejora en } \\
\text { socialización, vocalización, } \\
\text { rendimiento escolar, problemas } \\
\text { de conducta, ansiedad y patrones } \\
\text { de sueño (valoración parental). }\end{array}$ \\
\hline $\begin{array}{l}\text { Ibric y } \\
\text { Hudspeth } \\
(2003)\end{array}$ & Caso único. & Chico, 8 años. Autismo. & $\begin{array}{l}40 \text { sesiones NF: reducir } \\
\text { theta-aumentar alpha. }\end{array}$ & - & $\begin{array}{l}\text { Reducción de conductas } \\
\text { agresivas, obsesiones y } \\
\text { movimientos involuntarios. } \\
\text { Mejora en patrones de sueño. }\end{array}$ \\
\hline
\end{tabular}


Tabla 1. Resumen de los estudios

\begin{tabular}{|c|c|c|c|c|c|}
\hline Autor & Diseño & Muestra & Tratamiento & Evaluaciones & Resultados \\
\hline $\begin{array}{l}\text { Thompson y } \\
\text { Thompson } \\
\text { (2003) }\end{array}$ & Serie de casos. & $\begin{array}{l}60 \text { casos. } 5-51 \text { años. } \\
\text { Diagnóstico: Autismo } \\
\text { de alto funcionamiento. }\end{array}$ & $\begin{array}{l}\text { 40-100 sesiones NF: } \\
\text { protocolo personalizado } \\
\text { (QEEG-guided). }\end{array}$ & $\begin{array}{l}\text { EEG. Escalas de } \\
\text { valoración parental y } \\
\text { profesores. Test de } \\
\text { inteligencia. } \\
\text { Rendimiento } \\
\text { académico. Test de } \\
\text { atención sostenida. }\end{array}$ & $\begin{array}{l}\text { Cambios en EEG. Mejora de } \\
\text { puntuación test de inteligencia. } \\
\text { Mejora en interacción social } \\
\text { (valoración parental). }\end{array}$ \\
\hline Linden (2004) & Serie de casos. & $\begin{array}{l}15 \text { casos. 5-15 años. } \\
\text { Diagnóstico: Autismo o } \\
\text { Asperger. }\end{array}$ & $\begin{array}{l}\text { 20-50 sesiones NF: } \\
\text { QEEG-guided. }\end{array}$ & $\begin{array}{l}\text { QEEG. Escalas de } \\
\text { valoración parental y de } \\
\text { profesores (TDAH y } \\
\text { Autismo). CARS. IVA. } \\
\text { Test de atención } \\
\text { sostenida. }\end{array}$ & $\begin{array}{l}\text { Cambios en EEG. Mejoras en } \\
\text { Test de Atención Sostenida. } \\
\text { Reducción de inatención e } \\
\text { hiperactividad (valoración } \\
\text { parental/de profesores). Mejora } \\
\text { en comunicación y socialización } \\
\text { (CARS). Reducción de } \\
\text { medicación estimulante. }\end{array}$ \\
\hline $\begin{array}{l}\text { Coben y } \\
\text { Hudspeth } \\
(2006)\end{array}$ & $\begin{array}{l}\text { Comparación entre } \\
\text { grupos ( } 2 \text { grupos } \\
\text { experimentales). } \\
\text { Asignación NO } \\
\text { aleatoria. }\end{array}$ & $\begin{array}{l}\mathrm{N}=14 \text { (7-7). } \\
\text { Diagnóstico: Autismo. } \\
\text { Con exceso de "mu" y } \\
\text { déficit en su supresión. }\end{array}$ & $\begin{array}{l}20 \text { sesiones NF: } \\
\text { entrenamiento de } \\
\text { coherencia zonas } \\
\text { centrales-periféricas de } \\
\text { Córtex Frontal }(\mathrm{N}=7) 0 \\
\text { montaje bipolar } \\
\text { interhemisférico }(\mathrm{N}=7) \text {. }\end{array}$ & $\begin{array}{l}\text { QEEG. Pruebas } \\
\text { neuropsicológicas } \\
\text { (GADS) de pragmática, } \\
\text { interacción social, } \\
\text { funciones ejecutivas, } \\
\text { habilidades } \\
\text { visoespaciales y } \\
\text { atención. }\end{array}$ & $\begin{array}{l}\text { Cambios en EEG. Mejoras en } \\
\text { atención, funciones ejecutivas, } \\
\text { habilidades viso-espaciales, } \\
\text { interacción social, pragmática. } \\
\text { Aumento en supresión de mu } \\
\text { (solo en el grupo "coherencia"). }\end{array}$ \\
\hline Coben (2007) & $\begin{array}{l}\text { Grupo control (lista de } \\
\text { espera). Método de } \\
\text { asignación no } \\
\text { especificado. }\end{array}$ & $\begin{array}{l}\mathrm{N}=50 \text { ( } 25 \text { control- } 25 \\
\mathrm{NF}) \text {. Diagnóstico: } \\
\text { Autismo. }\end{array}$ & $\begin{array}{l}20 \text { sesiones NF: } \\
\text { entrenamiento de } \\
\text { coherencia áreas } \\
\text { posteriores del } \\
\text { hemisferio derecho con } \\
\text { hipocoherencia. }\end{array}$ & $\begin{array}{l}\text { QEEG. Pruebas } \\
\text { neuropsicológicas de } \\
\text { procesamiento de caras. } \\
\text { Escalas de valoración } \\
\text { parental (déficit en } \\
\text { habilidades sociales). }\end{array}$ & $\begin{array}{l}\text { Cambios en EEG. Mejoras en } \\
\text { procesamiento de caras/ } \\
\text { procesamiento emocional y } \\
\text { habilidades sociales. }\end{array}$ \\
\hline $\begin{array}{l}\text { Coben y } \\
\text { Padolsky } \\
(2007)\end{array}$ & $\begin{array}{l}\text { Grupo control (lista de } \\
\text { espera). Asignación NO } \\
\text { aleatoria. }\end{array}$ & $\begin{array}{l}\mathrm{N}=49 \text { ( } 12 \text { control-37 } \\
\mathrm{NF}) \text {. Diagnóstico: } \\
\text { Autismo. }\end{array}$ & $\begin{array}{l}20 \text { sesiones NF: } \\
\text { reducción de } \\
\text { hipercoherencia. }\end{array}$ & $\begin{array}{l}\text { QEEG. ATEC. Medidas } \\
\text { neuropsicológicas de } \\
\text { atención, habilidades } \\
\text { viso-perceptivas, } \\
\text { lenguaje y funciones } \\
\text { ejecutivas. }\end{array}$ & $\begin{array}{l}\text { Reducción de hipercoherencia } \\
\text { (EEG). Reducción de } 49 \% \text { en } \\
\text { puntuación de ATEC. Mejoras en } \\
\text { atención, habilidades viso- } \\
\text { perceptivas, lenguaje y funciones } \\
\text { ejecutivas. }\end{array}$ \\
\hline $\begin{array}{l}\text { Pineda et al. } \\
\text { (2008) }\end{array}$ & $\begin{array}{l}\text { Grupo control (placebo: } \\
\text { feedback EMG). } \\
\text { Asignación aleatoria. } \\
\text { Diseño ciego. }\end{array}$ & $\begin{array}{l}\mathrm{N}=8 \text { ( } 3 \text { placebo- } 5 \mathrm{NF}) \text {. } \\
\text { Diagnóstico: Autismo } \\
\text { de alto funcionamiento. }\end{array}$ & $\begin{array}{l}30 \text { sesiones NF: } \\
\text { controlar mu en C4 + } \\
\text { feedback EMG de los } \\
\text { músculos del trapecio } \\
\text { (evita artefactos. }\end{array}$ & $\begin{array}{l}\text { QEEG. Índice de } \\
\text { supresión de mu (MSI). } \\
\text { TOVA. Apraxia } \\
\text { Imitation Scale. ATEC. }\end{array}$ & $\begin{array}{l}\text { Reducción de coherencia C3-C4 } \\
\text { en todas las bandas de frecuencia. } \\
\text { Reducción de la amplitud de mu } \\
\text { en C3-C4. Mejora en TOVA: } \\
\text { reducción del } 70 \% \text { en puntuación } \\
\text { total de TDAH y errores de } \\
\text { comisión. Mejora en dimensión } \\
\text { "conciencia sensorial-cognitiva" } \\
\text { de ATEC. }\end{array}$ \\
\hline $\begin{array}{l}\text { Pineda et al. } \\
\text { (2008) }\end{array}$ & $\begin{array}{l}\text { Grupo control (placebo: } \\
\text { feedback EMG). } \\
\text { Asignación aleatoria. } \\
\text { Diseño doble ciego. }\end{array}$ & $\begin{array}{l}\mathrm{N}=19 \text { (10 placebo-9 } \\
\mathrm{NF}) \text {. Diagnóstico: } \\
\text { Autismo de alto } \\
\text { funcionamiento. }\end{array}$ & $\begin{array}{l}30 \text { sesiones NF: } \\
\text { controlar mu en C4 } \\
\text { (puntos de corte } \\
\text { QEEG-Guided) }+ \\
\text { feedback EMG de los } \\
\text { músculos del trapecio. }\end{array}$ & $\begin{array}{l}\text { QEEG. Índice de } \\
\text { supresión de mu (MSI). } \\
\text { TOVA. Apraxia } \\
\text { Imitation Scale. ATEC. }\end{array}$ & $\begin{array}{l}\text { Reducción de coherencia C3-C4 } \\
\text { en todas las bandas de frecuencia. } \\
\text { Mejoras en supresión de mu. } \\
\text { Mejora en TOVA: reducción de la } \\
\text { puntuación total de TDAH y de } \\
\text { errores de omisión (reducción de } \\
\text { errores de comisión tanto en } \\
\text { grupo control como } \\
\text { experimental). Mejora en todas } \\
\text { las dimensiones de ATEC (sin } \\
\text { diferencias significativas con } \\
\text { grupo control en la dimensión } \\
\text { "conciencia sensorial-cognitiva"). }\end{array}$ \\
\hline
\end{tabular}


Tabla 1. Resumen de los estudios

\begin{tabular}{|c|c|c|c|c|c|}
\hline Autor & Diseño & Muestra & Tratamiento & Evaluaciones & Resultados \\
\hline $\begin{array}{l}\text { Kouijzer et al. } \\
(2009 b)\end{array}$ & $\begin{array}{l}\text { Grupo control (lista de } \\
\text { espera). Asignación NO } \\
\text { aleatoria. Seguimiento } \\
12 \text { meses. }\end{array}$ & $\begin{array}{l}\mathrm{N}=14 \text { (7 control-7 NF). } \\
\text { Diagnóstico: Trastorno } \\
\text { Generalizado del } \\
\text { Desarrollo No } \\
\text { Especificado (TGD- } \\
\text { NE). }\end{array}$ & $\begin{array}{l}40 \text { sesiones NF: reducir } \\
\text { theta-aumentar beta en } \\
\text { C4. }\end{array}$ & $\begin{array}{l}\text { QEEG. Evaluación de } \\
\text { funciones ejecutivas: } \\
\text { control de la atención } \\
\text { (CPT del CNSVS y } \\
\text { TOSSA), flexibilidad } \\
\text { cognitiva (VBM y VIM de } \\
\text { CNSVS, TMT y MCST), } \\
\text { planificación (TOL) y } \\
\text { velocidad y eficacia (SDC } \\
\text { de CNSVS). Instrumento } \\
\text { de valoración parental: } \\
\text { comunicación } \\
\text { (CCC-2-NL) y síntomas } \\
\text { centrales del autismo } \\
\text { (AUTI-R). }\end{array}$ & $\begin{array}{l}\text { Reducción de theta, incremento } \\
\text { de beta y reducción de delta. } \\
\text { Mejoras en control de la atención } \\
\text { (CPT, TOSSA), flexibilidad } \\
\text { cognitiva (TMT, MCTS), } \\
\text { planificación (TOL), CCC-2-NL } \\
\text { y AUTI-R. Todos los cambios se } \\
\text { mantienen tras } 12 \text { meses. }\end{array}$ \\
\hline Coben (2009) & $\begin{array}{l}\text { Grupo control (lista de } \\
\text { espera). }\end{array}$ & $\begin{array}{l}\mathrm{N}=110(25 \text { control- } 85 \\
\mathrm{NF})\end{array}$ & $\begin{array}{l}74 \text { sesiones (media) NF: } \\
\text { protocolo no } \\
\text { especificado. }\end{array}$ & $\begin{array}{l}\text { QEEG. Pruebas } \\
\text { neuropsicológicas de } \\
\text { atención, habilidades } \\
\text { viso-perceptivas, } \\
\text { lenguaje y funciones } \\
\text { ejecutivas. ATEC. } \\
\text { Comparación de } \\
\text { mejoras entre niveles de } \\
\text { gravedad del trastorno. }\end{array}$ & $\begin{array}{l}\text { Reducción de síntomas ATEC. } \\
\text { Mejora de } 48 \% \text { en habilidades } \\
\text { viso-perceptivas, } 47 \% \text { en } \\
\text { lenguaje, } 56 \% \text { en atención y } 48 \% \\
\text { en funciones ejecutivas. Sin } \\
\text { diferencias significativas entre } \\
\text { distintos niveles de gravedad. }\end{array}$ \\
\hline $\begin{array}{l}\text { Kouijzer et al. } \\
\text { (2010) }\end{array}$ & $\begin{array}{l}\text { Grupo control (lista de } \\
\text { espera). Seguimiento } 6 \\
\text { meses. }\end{array}$ & $\begin{array}{l}\text { N=20 (10 control-10 } \\
\text { NF). Diagnóstico: } \\
\text { Autismo, Asperger o } \\
\text { TGD-NE. }\end{array}$ & $\begin{array}{l}40 \text { sesiones NF: reducir } \\
\text { theta (localización y } \\
\text { puntos de corte } \\
\text { QEEG-Guided). }\end{array}$ & $\begin{array}{l}\text { QEEG. Cuestionarios de } \\
\text { valoración parental y de } \\
\text { profesores: comunicación, } \\
\text { interacción y } \\
\text { comportamientos } \\
\text { estereotipados (SCQ, } \\
\text { SRS, CCC-2). Evaluación } \\
\text { de funciones ejecutivas: } \\
\text { control de la atención } \\
\text { (TOSSA), flexibilidad } \\
\text { cognitiva (TMT, MCST), } \\
\text { planificación (TOL), } \\
\text { rapidez y eficacia } \\
\text { (Stroop). }\end{array}$ & $\begin{array}{l}\text { Reducción de theta. Correlación } \\
\text { significativa entre reducción de } \\
\text { theta y mejoras en SCQ. Mejoras } \\
\text { según valoración parental (pero } \\
\text { no según profesores) en } \\
\text { interacción social -reciprocidad-, } \\
\text { comunicación -semántica, } \\
\text { coherencia, inicio de la } \\
\text { comunicación y comunicación no } \\
\text { verbal- (SCQ y CCC-2). Ligera } \\
\text { mejora en flexibilidad cognitiva. } \\
\text { Los cambios se mantienen tras } 6 \\
\text { meses. }\end{array}$ \\
\hline $\begin{array}{l}\text { Thompson et } \\
\text { al. (2010b) }\end{array}$ & Serie de casos. & $\begin{array}{l}150 \text { casos. } 117 \text { niños, } \\
30 \text { adolescentes y } 12 \\
\text { adultos. Diagnóstico: } \\
141 \text { Asperger y } 9 \\
\text { Autismo. }\end{array}$ & $\begin{array}{l}\text { 40-60 sesiones NF } \\
\text { (protocolo QEEG- } \\
\text { Guided) }+ \\
\text { Entrenamiento de } \\
\text { megacognitivo }+ \\
\text { Feedback de } \\
\text { temperatura, ritmo } \\
\text { cardiaco y actividad } \\
\text { electrodérmica (solo } \\
\text { adultos y adolescentes). }\end{array}$ & - & $\begin{array}{l}\text { Mejoras en los resultados de las } \\
\text { Escalas de Inteligencia de } \\
\text { Wechsler, WRAT-3, TOVA, IVA y } \\
\text { en síntomas centrales del } \\
\text { Síndrome de Asperger. }\end{array}$ \\
\hline $\begin{array}{l}\text { Kouijzer et al. } \\
\text { (2013) }\end{array}$ & $\begin{array}{l}\text { Comparación entre } \\
\text { grupos ( } 2 \text { grupos } \\
\text { experimentales) con } \\
\text { grupo control (lista de } \\
\text { espera). Asignación } \\
\text { aleatoria. Diseño ciego. } \\
\text { Seguimiento } 6 \text { meses. }\end{array}$ & $\begin{array}{l}\mathrm{N}=38 \text { (13 control, } 13 \\
\text { NF, } 12 \text { Feedback } \\
\text { Electrodérmico })\end{array}$ & $\begin{array}{l}40 \text { sesiones NF } \\
\text { (protocolo QEEG } \\
\text { guided) o Feedback } \\
\text { Electrodérmico. }\end{array}$ & $\begin{array}{l}\text { QEEG. Síntomas } \\
\text { centrales de los } \\
\text { trastornos del espectro } \\
\text { autista (SCQ), mejoría } \\
\text { clínica (CGI), } \\
\text { flexibilidad cognitiva } \\
\text { (TMT), inhibición } \\
\text { (Stroop), planificación } \\
\text { (TOL), atención } \\
\text { (TOSSA), memoria de } \\
\text { trabajo (subtest "Digit } \\
\text { Span" del WISC-III-NL). }\end{array}$ & $\begin{array}{l}\text { Mejoras en flexibilidad cognitiva. } \\
\text { Sin resultados significativos en el } \\
\text { resto de evaluaciones (ninguna } \\
\text { mejora o mejora sin diferencias } \\
\text { con el grupo de Feedback } \\
\text { Electrodérmico). }\end{array}$ \\
\hline
\end{tabular}

NF: Neurofeedback. TOVA: Test of Variables of Attention. SMR: Sensorimotor Rhythm. QEEG: EEG cuantitativo. ATEC: Autism Treatment Evaluation Checklist. CARS: Chilhood Autism Rating Scale. IVA: Integrated Visual and Auditory continuous performance test. GADS: Gilliam Asperger's Disorder Scale. CNSVS: Batería neuropsicológica CNS Vital Signs (CPT-Continuous Performance Test, VBM-Verbal Memory, VIB-Visual Memory, SDC-Symbol Digit Coding). TOSSA: Test of Sustained Selective Attention. TMT: Trial Making Test. MCST: Milwaukee Card Sorting Test. TOL: Tower of London. CCC-2-NL/CCC-2: Children's Communication Checklist. AUTI-R: Escala de screening para identificar niños con autismo. SCQ: Social Comunication Questionnaire. SRS:Social Responsiveness Scale. 
rosa, tal como puede observarse en la Tabla 1, cuyos resultados muestran también la normalización de los niveles basales de Mu y de su supresión en condiciones de imitación y observación de movimiento.

Otros dos estudios controlados se centran en la normalización de los patrones de hiper-/hipo- coherencia. En uno de ellos, Coben (2007) presenta los resultados de 20 sesiones de entrenamiento de coherencia empleando protocolos individualizados, y encuentra mejoras significativas en coherencia y normalización de los patrones de theta en diversas áreas cerebrales, asociados a mejoras en el procesamiento visual, de caras y de emociones (Coben et al., 2010a). Por otra parte, Coben y Padolsky (2007) se centran en reducir la hipercoherencia entre regiones frontales posteriores y áreas temporales anteriores usando protocolos individualizados, y sus resultados muestran una reducción significativa de la misma en un $76 \%$ de los participantes del grupo experimental. Además, dichos cambios se asocian a mejoras clínicamente significativas en la sintomatología. No obstante, estos resultados deben interpretarse con cautela puesto que no se dispone de las medidas de EEG del grupo control para realizar comparaciones entre grupos (Holtmann, Steiner, Hohmann, Poutska, Banaschewski y Bölte, 2011).

Por último, existe un estudio controlado de reciente publicación cuyos resultados contradicen a los anteriores (Kouijzer, van Schie, Gerrits, Buitelaar y de Moor, 2013). Éste cuenta con un diseño bastante complejo, como puede observarse en la Tabla 1, en el que la muestra se divide en tres grupos que posteriormente se subdividen aun más para realizar el análisis de los datos, lo cual podría suponer una dificultad añadida de cara a hallar la significación estadística y explicar, al menos en parte, la ausencia de significatividad de sus resultados.

\section{Sintomas centrales de los trastornos del espectro autista (TEA)}

El objetivo prioritario de todos los estudios revisados es comprobar los efectos del neurofeeback sobre los síntomas centrales de los TEA (i.e. déficits en interacción o socialización, déficits en comunicación o lenguaje, conductas estereotipadas o intereses restringidos). De los 17 estudios, solo uno concluye que el neurofeeback no tiene ningún efecto, a excepción de éste todos encuentran mejoras significativas en al menos una de las áreas mencionadas.

Cowan y Markham (1994) presentaron el primer estudio de caso único de la aplicación de neurofeeback a una niña diagnosticada de Autismo de Alto Funcionamiento. El tratamiento perseguía una reducción de ondas lentas y un incremento de ondas rápidas en áreas centra- les y parietales, y entre sus resultados se encuentra una reducción de las estereotipias y una mejora en la socialización según valoración parental y de profesores (Coben et al., 2010a). En un segundo estudio de este tipo, empleando un protocolo de neurofeeback similar, Sichel, Fehmi y Goldstein (1995) describen de forma más minuciosa una serie de mejoras en todas las dimensiones definitorias de los TEA: según valoración parental el niño comenzó a mostrarse más comunicativo y cariñoso, a iniciar más interacciones con sus hermanos e involucrarse en juegos imaginativos, mejoró el contacto visual y comenzó a buscar la ayuda de otros, se redujeron sus conductas estereotipadas y el apego a objetos inusuales, además su logopeda reportó mejoras significativas en lenguaje. En la misma línea, entre los resultados del caso presentado por Ibric et al. (2003) se encuentran mejoras en la interacción (e.g contacto visual), comunicación y lenguaje (p.ej., claridad del lenguaje, aspectos prosódicos y comprensión) y reducción de las estereotipias, todo ello basado en la valoración parental y de los terapeutas. También el trabajo presentado por Linden (2004) encuentra mejoras en comunicación y socialización tras el tratamiento en todos los casos, empleando escalas de valoración estandarizadas para padres y profesores (Coben et al., 2010a). Resultados que se replican nuevamente en las tres series de casos presentadas por Thompson et al. $(1995 ; 2003 ; 2010 b)$, empleando en este caso protocolos individualizados y reuniendo una muestra considerablemente mayor, tal como puede observarse en la Tabla 1.

Por lo que respecta a los estudios controlados, Jarusiewicz (2002) publicó el primero, donde aplica protocolos de neurofeeback individualizados que tienen como resultado una reducción media del $26 \%$ de la sintomatología medida por la escala Autism Treatment Evaluation Checklist (ATEC), que incluye subescalas de comunicación y lenguaje, sociabilidad, conciencia sensorial y cognitiva, y conductas saludables; mientras que en el grupo control solo se redujo un 3\%. No obstante, a la hora de interpretar estos resultados hay que tener en cuenta ciertas deficiencias metodológicas del estudio, como la falta de especificación de los instrumentos empleados para el diagnóstico y de los métodos de asignación a los grupos (Holtmann et al., 2011). Sus resultados son replicados más tarde, empleando también la escala de valoración ATEC, por otros estudios controlados de mayor rigurosidad metodológica. Ejemplo de ello es el estudio desarrollado por Coben et al. (2007), en el que un $89 \%$ de los padres del grupo experimental reportaron una reducción media en los síntomas de un $40 \%$, mientras que un $83 \%$ de los padres del grupo control no reportan ningún cambio; además, los resultados en las escalas neuropsicoló- 
gicas aplicadas apoyaron las valoraciones parentales. En la misma línea, un estudio más amplio realizado por Coben (2009) presenta también cambios significativos en las puntuaciones de la escala ATEC, así como en valoraciones neuropsicológicas objetivas. En este último, además, se realizaron análisis estadísticos para comprobar si los niveles de gravedad del trastorno o déficit intelectual anteriores al tratamiento influyen sobre la efectividad del mismo, y se concluyó que el neurofeeback es efectivo independientemente de dichos factores. Un mayor peso, dada su metodología, adquieren los dos estudios conducidos por Pineda et al. (2008), cuyos resultados nuevamente apoyan estos resultados, encontrando mejoras significativas en el grupo experimental en todas las dimensiones de la escala ATEC. Por otra parte, otros dos estudios obtienen resultados similares, empleando en este caso pruebas de evaluación distintas a la escala ATEC. Así, Coben et al. (2006) comparan los efectos de dos protocolos de neurofeeback y encuentran mejoras en interacción social y pragmática en ambos grupos, medidas por la escala de valoración neuropsicológica Gilliam Asperger's Disorder Scale (GADS) (Coben et al., 2011b); y Coben (2007), por su parte, describe efectos positivos sobre las habilidades sociales según valoración parental, y sobre el procesamiento de caras según pruebas estandarizadas. Finalmente, dos de los tres estudios conducidos por Kouijzer et al. (2009b, 2010) encontraron también mejoras significativas en comunicación, interacción y conducta social, según los resultados de distintas escalas de valoración parental; aunque en el caso del segundo estudio no se hallaron cambios según las valoraciones de los profesores. En cuanto al tercer trabajo realizado por Kouzjier et al. (2013), nuevamente contradice todos los hallazgos anteriores, reportando una ausencia de mejoras en la sintomatología central de los TEA, evaluada mediante cuestionarios estandarizados de valoración parental.

\section{Atención y funciones ejecutivas}

Un tercer grupo de variables con gran presencia en los estudios, presente en 10 de los 17 estudios, son aquellas relacionadas con la atención y las funciones ejecutivas, encontrando todos ellos mejoras significativas en al menos uno de sus componentes. La lógica de inclusión de estas variables reside tanto en la eficacia del neurofeeback para incidir en ellas, que ha sido demostrada en los estudios realizados con población con TDAH, como en la frecuente presencia de síntomas comórbidos de dicho trastorno en la población con TEA y las similitudes en sus patrones de EEG (Ruggieri, 2006). En esta línea, son muchos los estudios que analizan los perfiles aten- cionales de las personas con TEA y sus déficits, encontrando, a pesar de la variabilidad individual, resultados que apuntan la existencia de problemas a nivel de activación y modulación del foco atencional (López-Frutos, Stillo, Tripicchio y Campos, 2011). Además, cabe destacar la importancia de dichos déficits dado que podrían estar potenciando las dificultades de las personas con TEA en los intercambios sociales, por lo que debería considerarse la inclusión de variables atencionales en los programas de intervención dirigidos a esta población (de la Iglesia y Olivar, 2007; de la Iglesia y Olivar, 2008).

Cowan et al. (1994) encontraron mejoras en las medidas de inatención, impulsividad y variabilidad atencional, que además se mantuvieron dos años después del tratamiento. Los resultados positivos se repiten en el estudio de Linden (2004) y en la serie de casos presentada por Thompson et al. (2010b), evaluados mediante test de atención y escalas de valoración parental y de profesores. Por su parte, los dos estudios publicados por Pineda et al. (2008) encuentran resultados positivos en la puntuación general del Test of Variables of Attention (TOVA) y una reducción significativa de los errores por omisión en el grupo experimental, mientras que el resto de variables no muestran diferencias entre éste y el grupo que recibió el placebo. En el estudio conducido por Coben et al. (2007) se obtienen resultados positivos en atención, percepción visual y funciones ejecutivas; y en otro de sus trabajos (Coben, 2009) se describen, con mayor precisión, mejoras de un $43 \%$ en percepción visual, un $56 \%$ en habilidades atencionales y un $48 \%$ en funciones ejecutivas (Coben et al., 2011b). Por último, todos los estudios de Kouijzer et al. (2009b, 2010, 2013) encuentran mejoras en al menos una de las áreas evaluadas. El primero describe mejoras significativas en atención selectiva, atención sostenida y capacidad de inhibición de respuestas motoras y verbales en un test de atención sostenida y selectiva, reducción de interferencias en el test de Stroop, y una mejora en la capacidad de planificación y flexibilidad cognitiva. Sin embargo, en los dos trabajos restantes los resultados indican una influencia menor del neurofeeback en estas variables, reportan mejoras en flexibilidad cognitiva pero el resto de aspectos evaluados (p.ej., control atencional, planificación, etc.) permanecen sin cambios o bien no muestran diferencias con el grupo control.

\section{Otros resultados relevantes}

En algunos estudios se describen otros posibles efectos positivos del neurofeeback sobre otros síntomas o características inespecíficas de los TEA. Entre ellos, varios estudios reportan mejoras en los patrones de sueño 
(Ibric et al., 2003; Jarusiewicz, 2002; Sichel et al., 1995), reducción de las manifestaciones de ansiedad (Jarusiewicz, 2002; Sichel et al., 1995), reducción de las conductas disruptivas o rabietas (Ibric et al., 2003; Jarusiewicz, 2002) y mejoras en rendimiento académico o en pruebas de inteligencia (Jarusiewicz, 2002; Thompson et al., 2003; 2010b). Además, dos estudios describen en algunos casos una reducción de la cantidad o dosis de fármacos empleados, especialmente de tipo estimulantes (Linden, 2004; Thompson et al., 2010b). Por último, en los tres estudios conducidos por Kouijzer et al. (2009a, $2010,2013)$ se llevan a cabo seguimientos de los resultados tras 6-12 meses, encontrando en todos ellos que los cambios se mantienen a largo plazo.

\section{Discusión}

En el presente trabajo se ha realizado una revisión sistemática con el objetivo de averiguar si el neurofeeback es o no un tratamiento eficaz para población con TEA. A pesar de sus posibles limitaciones, como la inclusión de todos los estudios independientemente de su rigurosidad metodológica, la ausencia de análisis cuantitativos o los posibles sesgos introducidos por la propia interpretación de la información, esta revisión aporta una revisión amplia y reciente de la literatura disponible en la materia, con el aliciente de ser la única en lengua española.

A modo de síntesis de los resultados, la variable dependiente más estudiada la constituyen los síntomas centrales de los TEA, en la que todos los estudios menos uno encuentran mejoras significativas tras el tratamiento, entre las cuales destacan las mejoras en interacción y socialización, seguidas de mejoras en comunicación y lenguaje, y por último de reducciones de conductas estereotipadas. Entre estos estudios se incluyen bastantes con una rigurosidad metodológica considerable que emplean grupos control, tratamientos placebo, diseños ciego o doble ciego; cuyos resultados son coherentes con los obtenidos tanto en estudios no controlados como en estudios de caso. Una segunda variable con bastante presencia en los estudios son los cambios en el EEG basal, destacando las reducciones de theta y aumentos de beta, alfa o SMR, seguidos de las mejoras en la regulación de las ondas $\mathrm{Mu}$, y reducciones en la hipercoherencia en diversas áreas cerebrales. Entre los estudios que contemplan dicha variable, solo dos son series de casos, mientras que otros nueve son estudios controlados, algunos de los cuáles además emplean diseños ciego o doble ciego. Otras variables a tener en cuenta son los déficits en atención y en funciones ejecutivas, descritas en conjunto por su gran relación funcional y anatómica. Son precisa- mente dichas variables las que presentan los resultados más consistentes, ya que los 10 trabajos que las estudian encuentran algún tipo de mejora en ellas, a saber: mejoras en pruebas de atención sostenida y atención selectiva, así como en flexibilidad cognitiva y planificación, y reducciones de la impulsividad. Es por ello que algunos investigadores, como Holtmann et al. (2011), consideran que los efectos del neurofeeback en población con TEA podrían estar mediados por su comorbilidad sintomática con los TDAH. Entre estos 10 trabajos se encuentra un estudio de caso único, dos series de casos y 7 estudios controlados. Por último, algunos estudios encuentran indicios de que el neurofeeback podría mejorar, directa o indirectamente, sintomatología más inespecífica pero bastante frecuente en la población con TEA, como los trastornos del sueño, la ansiedad y las conductas disruptivas, o los déficits en el rendimiento académico/intelectual. Si bien, cabe indicar que dichos resultados provienen mayoritariamente de estudios de caso único o series de casos y de valoraciones parentales o de profesores con un importante grado de subjetividad.

Los datos disponibles hasta el momento no son suficientes para concluir de forma decisiva que el neurofeeback sea un tratamiento de elección para los TEA, puesto que la disparidad en los resultados y los déficits metodológicos presentes en algunos de los estudios no permiten confirmar que se trate de una alternativa sistemáticamente eficaz ni superior a otras. Dicha disparidad de resultados puede deberse a la amplia variabilidad entre los estudios (muestras, protocolos de tratamiento, diseños de investigación, etc.). Además de esto, otro gran inconveniente de muchos de los estudios disponibles hasta el momento es la ausencia de comprobaciones acerca de la correlación entre los cambios en EEG y las mejoras en la sintomatología descritas tras el tratamiento; hecho que limita la posibilidad de discernir si dichas mejoras son consecuencia directa o no de los efectos del neurofeeback sobre la actividad cerebral de los pacientes, que es, en última instancia, la hipótesis de base para el desarrollo de dicho tratamiento. Así pues, de cara a conseguir un mayor grado de certeza en nuestras conclusiones sería necesario realizar nuevas investigaciones en las que, además de incluir los datos de correlación mencionados previamente, se solucionen los déficits metodológicos presentes en los estudios de los que disponemos actualmente, empleando diseños controlados, muestras más amplias y exhaustivas o bien muestras más heterogéneas cuando éstas sean reducidas, diseños doble ciego y pruebas de evaluación estandarizadas para prevenir los sesgos introducidos por el investigador, etc.

No obstante, a pesar de todos los inconvenientes previamente mencionados, siguiendo los niveles de eviden- 
cia establecidos por la APA el tratamiento con neurofeeback para los TEA se consideraría como un tratamiento "probablemente eficaz" o "con apoyo experimental modesto", al contar con el apoyo de al menos un estudio bien controlado o varios estudios bien diseñados, incluyendo estudios de caso único; aunque cabría especificar que el apoyo es controvertido, dada la amplia variabilidad entre estudios.

Con todo, aunque no existe evidencia científica suficiente para afirmar que el neurofeeback pueda constituir el tratamiento de elección para los TEA, sí que puede concluirse con relativa certeza que es, como mínimo, un complemento adecuado para aplicar junto con otras intervenciones. Además, se trata de una técnica relativamente barata, no invasiva, sin efectos secundarios relevantes, flexible y fácil de adaptar a las necesidades de cada caso y cuyos efectos positivos se mantienen a largo plazo. Todas estas características hacen del neurofeeback una técnica prometedora cuya introducción en la práctica clínica habitual podría resultar muy interesante, y sobre la que sin duda merece la pena continuar investigando.

\section{Referencias}

Abarbanel, A. (1999). 12 - the neural underpinnings of neurofeedback training. In J. R. Evans, \& A. Abarbanel (Eds.), Introduction to quantitative EEG and neurofeedback (pp. 311340). San Diego: Academic Press. doi: http://dx.doi. org/10.1016/B978-012243790-8/50013-4

American Psychiatric Association. (2013). DSM-5: Diagnostic and statistical manual of mental disorders. Washington: American Psychiatric Association.

Bernier, R., Dawson, G., Webb, S., \& Murias, M. (2007). EEG mu rhythm and imitation impairments in individuals with autism spectrum disorder. Brain and Cognition, 64, 228-237. doi: http://dx.doi.org/10.1016/j.bandc.2007.03.004

Billeci, L., Sicca, F., Maharatna, K., Apicella, F., Narzisi, A., Campatelli, G., ... \& Muratori, F. (2013). On the application of quantitative EEG for characterizing autistic brain: A systematic review. Frontiers in Human Neuroscience, 7.

Burnette, C. P., Henderson, H. A., Inge, A. P., Zahka, N. E., Schwartz, C. B., \& Mundy, P. C. (2011). Anterior EEG asymmetry and the modifier model of autism. Journal of Autism and Developmental Disorders, 41, 1113-1124. doi: http://dx. doi.org/10.1007/s10803-010-1138-0

Cantor, D. S. (1999). 1 - an overview of quantitative EEG and its applications to neurofeedback. In J. R. Evans, \& A. Abarbanel (Eds.), Introduction to quantitative EEG and neurofeedback (pp. 3-27). San Diego: Academic Press. doi: http://dx.doi. org/10.1016/B978-012243790-8/50002-X

Cantor, D. S., Thatcher, R. W., Hrybyk, M., \& Kaye, H. (1986). Computerized EEG analyses of autistic children. Journal of Autism and Developmental Disorders, 16, 169-187. doi: http://dx.doi.org/10.1007/BF01531728
Centers for Disease Control and Prevention (CDC) (2012). Prevalence of autism spectrum disorders--autism and developmental disabilities monitoring network, 14 sites, united states, 2008. Morbidity and Mortality Weekly Report. Surveillance Summaries, 61, 1-19.

Chan, A. S., \& Leung, W. W. M. (2006). Differentiating autistic children with quantitative encephalography: A 3-month longitudinal study. Journal of Child Neurology, 21, 391-399.

Chan, A. S., Sze, S. L., \& Cheung, M. (2007). Quantitative electroencephalographic profiles for children with autistic spectrum disorder. Neuropsychology, 21, 74-81. doi: http://dx.doi. org/10.1037/0894-4105.21.1.74

Chez, M. G., Chang, M., Krasne, V., Coughlan, C., Kominsky, M., \& Schwartz, A. (2006). Frequency of epileptiform EEG abnormalities in a sequential screening of autistic patients with no known clinical epilepsy from 1996 to 2005. Epilepsy \& Behavior, 8, 267-271. doi: http://dx.doi.org/10.1016/j.yebeh.2005.11.001

Coben, R. (2007, September). Autistic spectrum disorder: A controlled study of EEG coherence training targeting social skill deficits. Presented at the 15 th annual conference of the International Society for Neurofeedback and Research, San Diego, California.

Coben, R. (2009). Efficacy of connectivity-guided neurofeedback for Autistic Spectrum Disorder: Controlled analysis of $75 \mathrm{ca}-$ ses with a 1 to 2 year follow-up. Journal of Neurotherapy, 13, 81.

Coben, R., \& Evans, J. (2011a). Preface. In R. Coben, \& J. R. Evans (Eds.), Neurofeedback and neuromodulation techniques and applications (pp. xvxvii). San Diego: Academic Press. doi: http://dx.doi.org/10.1016/B978-0-12-3822352.00019-6

Coben, R., \& Hudspeth, W. (2006, September). Mu-like rhythms in autistic spectrum disorder: EEG analyses and neurofeedback. Presented at the 14th Annual Conference of the International Society for Neuronal Regulation, Atlanta, Georgia.

Coben, R., \& Myers, T. E. (2008b). Connectivity theory of autism: Use of connectivity measures in assessing and treating autistic disorders. Journal of Neurotherapy, 12, 161-179. doi: http:// dx.doi.org/10.1080/10874200802398824

Coben, R., \& Myers, T. E. (2010b). The relative efficacy of connectivity guided and symptom based EEG biofeedback for autistic disorders. Applied Psychophysiology and Biofeedback, 35, 13-23. doi: http://dx.doi.org/10.1007/s10484-0099102-5

Coben, R., \& Padolsky, I. (2007). Assessment-guided neurofeedback for autistic spectrum disorder. Journal of Neurotherapy, 11, 5-23. doi: http://dx.doi.org/10.1300/J184v11n01_02

Coben, R., \& Wagner, L. A. (2011b). Chapter 6 - emerging empirical evidence supporting connectivity-guided neurofeedback for autistic disorders. In R. Coben, \& J. R. Evans (Eds.), Neurofeedback and neuromodulation techniques and applications (pp. 153-182). San Diego: Academic Press. doi: http://dx.doi. org/10.1016/B978-0-12-382235-2.00006-8

Coben, R., Clarke, A. R., Hudspeth, W., \& Barry, R. J. (2008a). EEG power and coherence in autistic spectrum disorder. Clinical Neurophysiology, 119, 1002-1009. doi: http://dx.doi.org/10.1016/j.clinph.2008.01.013 
Coben, R., Linden, M., \& Myers, T. E. (2010a). Neurofeedback for autistic spectrum disorder: A review of the literature. Applied Psychophysiology and Biofeedback, 35, 83-105. doi: http://dx.doi.org/10.1007/s10484-009-9117-y

Cornew, L., Roberts, T. P., Blaskey, L., \& Edgar, J. C. (2012). Resting-state oscillatory activity in autism spectrum disorders. Journal of Autism and Developmental Disorders, 42, 18841894. doi: http://dx.doi.org/10.1007/s10803-011-1431-6

Cowan, J., \& Markham, L. (1994, March). EEG biofeedback for the attention problems of autism: A case study. Presented at the 25th Annual Meeting of the Association for Applied Psychophysiology and Biofeedback, Atlanta, GA.

Dawson, G., Klinger, L. G., Panagiotides, H., Lewy, A., \& Castelloe, P. (1995). Subgroups of autistic children based on social behavior display distinct patterns of brain activity. Journal of Abnormal Child Psychology, 23, 569-583. doi: http://dx.doi. org/10.1007/BF01447662

De la Iglesia, M. y Olivar, J.S. (2008). Intervenciones sociocomunicativas en los trastornos del espectro autista de alto funcionamiento. Revista de Psicopatología y Psicología Clínica, 13, 1-19. doi: http://dx.doi.org/10.5944/rppc.vol.13.num.1.2008.4046

De la Iglesia, M. y Olivar, J.S. (2007). Entrenamiento en habilidades sociocomunicativas en los trastornos del espectro autista de alto funcionamiento. Revista de Psicopatología y Psicología Clínica, 12, 33-42. doi: http://dx.doi.org/10.5944/rppc. vol.12.num.1.2007.4032

Dorenbaum, D., Mencel, E., Blume, W. T., \& Fisman, S. (1987). EEG findings and language patterns in autistic children: Clinical correlations. The Canadian Journal of Psychiatry / La Revue Canadienne De Psychiatrie, 32, 31-34.

Gabard-Durnam, L., Tierney, A. L., Vogel-Farley, V., Tager-Flusberg, H., \& Nelson, C. A. (2013). Alpha asymmetry in infants at risk for autism spectrum disorders. Journal of Autism and Developmental Disorders. doi: http://dx.doi.org/10.1007/ s10803-013-1926-4

Hammond, D. C. (2007). What is neurofeedback? Journal of Neurotherapy, 10, 25-36. doi: http://dx.doi.org/10.1300/ J184v10n04_04

Holtmann, M., Steiner, S., Hohmann, S., Poustka, L., Banaschewski, T., \& Bölte, S. (2011). Neurofeedback in autism spectrum disorders. Developmental Medicine \& Child Neurology, 53, 986-993. doi: http://dx.doi.org/10.1111/j.1469-8749.2011.04043.x

Hughes, J. R., \& John, E. R. (1999). Conventional and quantitative electroencephalography in psychiatry. The Journal of Neuropsychiatry and Clinical Neurosciences, 11, 190-208.

Ibric, V. L., \& Hudspeth, W. (2003, February). QEEG and Roshi use in autism post-toxic encephalopathy-a case study. Presented at the 11th Annual Winter Brain Conference, Palm Springs, CA.

Jarusiewicz, B. (2002). Efficacy of neurofeedback for children in the autistic spectrum: A pilot study. Journal of Neurotherapy, 6, 39-49. doi: http://dx.doi.org/10.1300/J184v06n04_05

Kouijzer, M. E. J., de Moor, J. M. H., Gerrits, B. J. L., Buitelaar, J. K., \& van Schie, H. T. (2009a). Long-term effects of neurofeedback treatment in autism. Research in Autism Spectrum Disorders, 3, 496-501. doi: http://dx.doi.org/10.1016/j. rasd.2008.10.003

Kouijzer, M. E. J., de Moor, J. M. H., Gerrits, B. J. L., Congedo, M., \& van Schie, H. T. (2009b). Neurofeedback improves exe- cutive functioning in children with autism spectrum disorders. Research in Autism Spectrum Disorders, 3, 145-162. doi: http://dx.doi.org/10.1016/j.rasd.2008.05.001

Kouijzer, M. E. J., van Schie, H. T., de Moor, J. M. H., Gerrits, B. J. L., \& Buitelaar, J. K. (2010). Neurofeedback treatment in autism. preliminary findings in behavioral, cognitive, and neurophysiological functioning. Research in Autism Spectrum Disorders, 4, 386-399. doi: http://dx.doi.org/10.1016/j. rasd.2009.10.007

Kouijzer, M. E. J., van Schie, H. T., Gerrits, B. J. L., Buitelaar, J. K., \& de Moor, J. M. H. (2013). Is EEG-biofeedback an effective treatment in autism spectrum disorders? A randomized controlled trial. Applied Psychophysiology and Biofeedback, 38, 17-28. doi: http://dx.doi.org/10.1007/s10484-012-9204-3

Kropotov, J. D. (2009). Quantitative EEG, event-related potentials and neurotherapy. Amsterdam etc.: Elsevier.

Linden, M. (2004, August). Case studies of QEEG mapping and neurofeedback with autism. Presented at the 12th Annual Conference of the International Society for Neuronal Regulation, Fort Lauderdale, FL.

López-Frutos, J.M., Sotillo, M., Tripicchio, P. y Campos, R. (2011). Funciones atencionales de orientación espacial, alerta y control ejecutivo en personas con trastornos del espectro autista. Revista de Psicopatología y Psicología Clínica, 16, 101-112. doi: http://dx.doi.org/10.5944/rppc.vol.16.num.2.2011.10354

Lushchekina, E. A., Podreznaya, E. D., Lushchekin, V. S., \& Strelets, V. B. (2012). A comparative EEG study in normal and autistic children. Neuroscience and Behavioral Physiology, 42, 236-243. doi: http://dx.doi.org/10.1007/s11055-0129558-2

Newschaffer, C. J., Croen, L. A., Daniels, J., Giarelli, E., Grether, J. K., Levy, S. E., ... \& Windham, G. C. (2007). The epidemiology of autism spectrum disorders*. Annual Review of Public Health, 28, 235-258.

Oberman, L. M., Hubbard, E. M., McCleery, J. P., Altschuler, E. L., Ramachandran, V., \& Pineda, J. A. (2005). EEG evidence for mirror neuron dysfunction in autism spectrum disorders. Cognitive Brain Research, 24, 190-198. doi: http://dx.doi.org/10.1016/j.cogbrainres.2005.01.014

Oberman, L. M., McCleery, J. P., Hubbard, E. M., Bernier, R., Wiersema, J. R., Raymaekers, R., \& Pineda, J. A. (2013). Developmental changes in mu suppression to observed and executed actions in autism spectrum disorders. Social Cognitive and Affective Neuroscience, 8, 300-304. doi: http://dx.doi. org $/ 10.1093 /$ scan/nsr097

Orekhova, E. V., Stroganova, T. A., Nygren, G., Tsetlin, M. M., Posikera, I. N., Gillberg, C., \& Elam, M. (2007). Excess of high frequency electroencephalogram oscillations in boys with autism. Biological Psychiatry, 62, 1022-1029. doi: http:// dx.doi.org/10.1016/j.biopsych.2006.12.029

Palau-Baduell, M., Valls-Santasusana, A., Salvado-Salvado, B., \& Clofent-Torrento, M. (2013). Aportacion del electroencefalograma en el autismo. Revista de Neurologia, 56 Suppl 1, S35-43.

Pineda, J. A., Brang, D., Hecht, E., Edwards, L., Carey, S., Bacon, M., ... \& Rork, A. (2008). Positive behavioral and electrophysiological changes following neurofeedback training in children with autism. Research in Autism Spectrum Disorders, 2, 557-581. 
Posada-De la Paz, M., Ferrari-Arroyo, M. J., Tourino, E., \& Boada, L. (2005). Investigación epidemiológica en el autismo: una visión integradora. Revista de Neurologia, 40 Suppl 1, S191-8.

Rippon, G., Brock, J., Brown, C., \& Boucher, J. (2007). Disordered connectivity in the autistic brain: Challenges for the 'new psychophysiology'. International Journal of Psychophysiology, 63, 164-172. doi: http://dx.doi.org/10.1016/j.ijpsycho.2006.03.012

Ruggieri, V. L. (2006). Procesos atencionales y trastornos por deficit de atencion en el autismo. Revista de Neurologia, $42 \mathrm{Su}$ ppl 3, S51-6.

Sichel, A. G., Fehmi, L. G., \& Goldstein, D. M. (1995). Positive outcome with neurofeedback treatment in a case of mild autism. Journal of Neurotherapy, 1, 60-64. doi: http://dx.doi. org/10.1300/J184v01n01_08

Thompson, L., \& Thompson, M. (1995, May). Autism/Asperger's/ obnoxious child, 3 case histories: How we get positive results with complex ADD clients. Paper presented at the Annual Conference of the Society for Neuronal Regulation, Scottsdale, AZ.
Thompson, L., \& Thompson, M. (2003). Neurofeedback treatment for autistic spectrum disorders: Review of 60 cases-principles and outcome. Citation paper presented at the 34th Annual Meeting of the Association for Applied Psychophysiology and Biofeedback, Jacksonville, FL.

Thompson, L., Thompson, M., \& Reid, A. (2010a). Functional neuroanatomy and the rationale for using EEG biofeedback for clients with Asperger's syndrome. Applied Psychophysiology and Biofeedback, 35, 39-61. doi: http://dx.doi. org/10.1007/s10484-009-9095-0

Thompson, L., Thompson, M., \& Reid, A. (2010b). Neurofeedback outcomes in clients with Asperger's syndrome. Applied Psychophysiology and Biofeedback, 35, 63-81. doi: http://dx. doi.org/10.1007/s10484-009-9120-3

Yasuhara, A. (2010). Correlation between EEG abnormalities and symptoms of autism spectrum disorder (ASD). Brain \& Development, 32, 791-798. doi: http://dx.doi.org/10.1016/j.braindev.2010.08.010 